\title{
High promoter activity of cytokeratin-19 gene in cholangiocarcinoma
}

\author{
JIAN QIN $^{1}$, MIN WANG ${ }^{1}$, JUN QIN ${ }^{1}$, QU CAI $^{2}$ and ZHI-HAI PENG ${ }^{1}$ \\ ${ }^{1}$ Department of Surgery, First People's Hospital Affiliated to Shanghai Jiaotong University, \\ Shanghai 200080; ${ }^{2}$ Shanghai Institute of Digestive Surgery, Ruijin Hospital, \\ Shanghai Jiaotong University School of Medicine, Shanghai 200025, P.R. China
}

Received August 31, 2017; Accepted July 30, 2018

DOI: $10.3892 /$ mco. 2018.1700

\begin{abstract}
Conditionally replicating adenoviral vectors constructed with tumor-specific promoters (TSPs) offers a viable tool for the treatment of cholangiocarcinoma. The aim of the present study was to investigate cholangiocarcinoma-specific TSPs that remain active in adenoviral constructs in gene therapy. The mRNA expressions of cyclooxygenase-2, cytokeratin-19 (CK19), mucin-1, midkine and telomerase reverse transcriptase were determined in human cholangiocarcinoma cell lines, primary human hepatocytes and cholangiocytes using reverse transcription-quantitative polymerase chain reaction. The candidate promoters constructed in adenoviral vectors were analyzed for their activities in cholangiocarcinoma cell lines, primary human hepatocytes and cholangiocytes using dual-luciferase reporter assays. The mRNA expression of CK19 was markedly higher in the QBC939 cell line, indicating specificity to cholangiocarcinoma. Moreover, the promoter activity of CK19 in the adenoviral vector in infected cholangiocarcinoma cells was found to be significantly stronger compared with that in infected primary human hepatocytes and cholangiocytes. CK19 may be implicated in the pathogenesis of cholangiocarcinoma, as demonstrated by the stronger activity of its promoter, as well as the higher expression of mRNA in tumor cells. Therefore, the use of the promoter sequence of the CK19 gene may represent a potential tool in cholangiocarcinoma-specific adenoviral gene therapy.
\end{abstract}

\section{Introduction}

Cholangiocarcinoma is a malignant tumor originating in the biliary tract, with an extremely poor prognosis at the advanced

Correspondence to: Dr Zhi-Hai Peng, Department of Surgery, First People's Hospital Affiliated to Shanghai Jiaotong University, Shanghai 200080, P.R. China

E-mail: pengzh18@outlook.com

Key words: cholangiocarcinoma, tumor-specific promoter, cytokeratin-19, gene therapy stages. An estimated 40-70\% of cholangiocarcinomas arise near the bifurcation of the right and left hepatic ducts at the liver hilum, whereas $\sim 5-20 \%$ of the cases develop within the liver (1). The incidence of cholangiocarcinoma increases in association with the higher incidence of chronic inflammatory liver diseases $(2,3)$. Cholangiocarcinoma comprises $\sim 15 \%$ of all primary liver tumors; however, only a small proportion of patients with early-stage cholangiocarcinoma are considered suitable for curative surgical treatment (4). Thus, prolonging the survival of patients with cholangiocarcinoma remains challenging and new treatment approaches are urgently required.

Gene therapy is partially effective in the treatment of certain types of diseases, including cancer $(5,6)$. The application of gene therapy has been somewhat limited; however, with the advances in molecular biology, the clinical application of gene therapy may move forward. The advent of adenoviral vectors provided a potential tool for the application of gene-based therapy for treating solid tumors. A major disadvantage was that the adenoviral vector also infects other replicating cells besides tumor cells. To minimize the side effects in tumor gene therapy, a selective adenoviral vector that only infects tumor cells is the research focus of interest in this field. Tumor-specific promoters (TSPs) play important roles in viral replication in tumors and they may be used to restrict gene expression in tumorous lesions. Attempts to use TSPs of known hepatobiliary biomarker genes, such as cyclooxygenase-2 (COX-2), midkine (MK), mucin-1 (MUC1), human telomerase reverse transcriptase (hTERT) and the bile duct marker cytokeratin-19 (CK19), offer a novel approach to restricting gene expression only in cholangiocarcinoma cells (7-11). In addition, the expression of transduced genes does not occur in non-proliferating primary liver cells when adenoviral vector is implicated in gene therapy (12). Other studies have demonstrated the effectiveness of potential TSPs constructed in adenoviral vectors used in gene therapy in cholangiocarcinoma; however, the selection of different TSPs may yield inconsistent results $(13,14)$. The aim of the present study was to investigate cholangiocarcinoma-specific biomarker genes and their promoters, which are functional in cholangiocarcinoma cells but remain silent in non-tumorous primary human liver cells, in order to determine the value of the potential application of cholangiocarcinoma-specific TSP in gene therapy. 


\section{Materials and methods}

Cell lines and primary cells. The human cholangiocarcinoma cell line QBC939 was provided by Hanbio Biotechnology Co., Ltd. (Shanghai, China). The normal human hepatic cell line LO2 was obtained from the American Type Culture Collection (Manassas, VA, USA). The cells were cultured in $10 \%$ fetal calf serum Dulbecco's modified Eagle's medium with $5 \% \mathrm{CO}_{2}$ at $37^{\circ} \mathrm{C}$. Human primary cholangiocytes were isolated from normal liver tissue from patients undergoing liver transplantation, and are commercially available from Wuhan PriCells Biomedical Technology Co., Ltd. (Wuhan, China). The cells were maintained in culture according to the manufacturer's instructions.

Reverse transcription-quantitative polymerase chain reaction $(R T-q P C R)$. Total RNA was isolated using TRIzol reagent (Invitrogen; Thermo Fisher Scientific, Carlsbad, CA, USA) and first-strand cDNA was synthesized using the Omniscript RT kit (Qiagen, Inc., Valencia, CA, USA). RT-qPCR was performed with SYBR dye on LightCycler 480 (Roche, Ltd., Basel, Switzerland) in triplicate, using the following cycling conditions: Denaturation at $95^{\circ} \mathrm{C}$ for $5 \mathrm{~min}$, followed by 50 cycles of amplification at $95^{\circ} \mathrm{C}$ for $10 \mathrm{sec}$ and extension at $60^{\circ} \mathrm{C}$ for $20 \mathrm{sec}$, melting at $95^{\circ} \mathrm{C}$ for $10 \mathrm{sec}$, followed by $10 \mathrm{sec}$ at $60^{\circ} \mathrm{C}$, and cooling at $40^{\circ} \mathrm{C}$ for $30 \mathrm{sec}$. The sequences of the primers of the genes of interest are listed in Table I. The $\Delta \Delta \mathrm{Cq}$ method was used for data analysis and the results were expressed in arbitrary units and normalized with GAPDH.

Recombinant adenoviral vectors. Wild-type adenoviral vector pAdeno and shuttle plasmid pShuttle2-Basic were provided by Hanbio Biotechnology Co., Ltd. (Shanghai, China). Plasmid pLuc-MCS containing firefly luciferase was purchased from Agilent Technologies (Santa Clara, CA, USA). The human CK19 promoter (-740-1-bp; GenBank accession no. AB045973) was amplified from human genomic DNA using the following primers: Forward: 5'-AAACCGCTCGAGCCTGTAATCCCA GCACTTTG-3'; reverse: 5'-CGGGGTACCGGCGAGGCG GAGCACGGAC-3'.

Ad-CK19-Luc, a recombinant adenoviral vector containing a luciferase gene driven by the CK19 promoter, was constructed as follows: The 2,700-bp BamHI fragment from pLuc-MCS, which contains cDNA for Luc, was ligated to the BamHI site of pShuttle2-Basic to yield pShuttle2-MCS-Luc; the 740-bp XhoI/KpnI fragment from the CK19 promoter was ligated to the $X h o \mathrm{I} / K p n \mathrm{I}$ site of pShuttle2-MCS-Luc to yield pShuttle-CK19-Luc; the I-CeuI/I-SceI fragment from the CK19 promoter and Luc from pShuttle-CK19-Luc were inserted at the I-CeuI/I-SceI site of pAdeno to yield the Ad-CK19-Luc. Adenoviruses were propagated in HEK293 cells and the viral titer $(\mathrm{PFU} / \mathrm{ml})$ was determined. The propagated viruses were purified using $\mathrm{CsCl}$ gradient centrifugation, dialyzed against $10 \%$ glycerol PBS and stored at $-80^{\circ} \mathrm{C}$.

Promoter activity assay. Cells $\left(2 \times 10^{5}\right)$ were cultured in 6 -well plates. After $12 \mathrm{~h}$, the cells were infected with Ad-cytomegalovirus (CMV)-Luc and Ad-CK19-Luc at a multiplicity of infection rate of 1 and 10 , respectively. The medium was replaced with fresh medium $2 \mathrm{~h}$ after infection.
Luciferase activity was determined with the Dual-Luciferase Reporter Assay system (Promega, Madison, WI, USA) $48 \mathrm{~h}$ after transduction, according to the manufacturer's protocol. The efficiency of transduction was evaluated in all cell types with Ad-CMV-Luc to ensure non-saturating multiplicity of infection. The data were presented as value relative to that of the constitutively active CMV promoter.

Statistical analysis. The statistical analysis was performed using the SPSS v14.0 statistical software package (SPSS, Inc., Chicago, IL, USA). Statistical differences were analyzed with the Student's t-test (unpaired, two-tailed). A P-value of $<0.05$ was considered to indicate statistically significant differences between groups for all experiments.

\section{Results}

Analysis of mRNA levels of biomarkers for high expression in cholangiocarcinoma. To assist with the selection of cholangiocarcinoma-specific promoters, the best specific biomarker for cholangiocarcinoma were first screened out. The mRNA expression of the COX-2, CK19, MK, MUC1 and hTERT genes in human cholangiocarcinoma cell lines, primary human hepatocytes and cholangiocytes were measured. The results demonstrated that CK19 exhibited the best tumor specificity, with median mRNA expression ratios of $128.9100 \pm 27.18$, $0.0052 \pm 0.0013$ and $1.0020 \pm 0.073$ in cholangiocarcinoma cell lines, normal primary human hepatocytes and cholangiocytes, respectively ( $\mathrm{P}<0.001$; Fig. 1$)$. The median expression in cholangiocarcinoma cell lines was 24,600-fold higher compared with that in normal primary human hepatocytes, and 130 -fold higher compared with that in normal primary human cholangiocytes. However, the mRNA expression of COX-2, MK, MUC1 and hTERT did not show a relevance to cholangiocarcinoma. The mRNA expression levels of MUC1, hTERT and COX-2 were significantly higher in primary human hepatocytes compared with those in the cholangiocarcinoma cell line $(\mathrm{P}<0.01)$. The mRNA expression of MK was high in primary human cholangiocytes and cholangiocarcinoma cell lines, but without significant differences between the two types of cells $(\mathrm{P}>0.05)$. Since the mRNA expression of CK19 was cholangiocarcinoma-specific, the CK19 promoter was selected for further analyses.

TSP activity assays. To implement tumor promoters in gene therapy, we investigated whether the CK19 promoter retained tumor specificity when constructed in an adenovirus vector. Therefore, the adenoviral reporter vector driving luciferase from the CK19 promoter fragment (-740-1-bp) was constructed. The activities of the adenoviral promoter constructs were determined in the infected cholangiocarcinoma cells, primary human hepatocytes and cholangiocytes. The data were presented as the value relative to that of the constitutively active CMV promoter. As shown in Fig. 2, transduction with Ad-CK19-Luc resulted in the highest luciferase activity in cholangiocarcinoma cells as compared with that in primary human hepatocytes and cholangiocytes. The median activity in QBC939 cells was $0.473 \pm 0.017$, which was significantly higher compared with that in primary human hepatocytes $(0.123 \pm 0.007)$ and cholangiocytes $(0.006 \pm 0.001)(\mathrm{P}<0.001)$. 
Table I. Primer sequences used in reverse transcription-quantitative polymerase chain reaction.

\begin{tabular}{lll}
\hline Genes & \multicolumn{1}{c}{ Forward primers $\left(5^{\prime}\right.$ to $\left.3^{\prime}\right)$} & Reverse primers $\left(5^{\prime}\right.$ to $\left.3^{\prime}\right)$ \\
\hline COX-2 & CCAGTATAAGTGCGATTGTACCC & TCAAAAATTCCGGTGTTGAGCA \\
MK & CGCGGTCGCCAAAAAGAAAG & TACTTGCAGTCGGCTCCAAAC \\
MUC1 & TCAGTGCCGCCGAAAGAAC & GCTCATAGGATGGTAGGTATCCC \\
hTERT & ATTGGAATCAGACAGCACTTGAA & TCCCACGACGTAGTCCATGTT \\
CK19 & ACCAAGTTTGAGACGGAACAG & CCCTCAGCGTACTGATTTCCT
\end{tabular}

COX-2, cyclooxygenase-2; MK, midkine; MUC1, mucin-1; hTERT, human telomerase reverse transcriptase; CK19, cytokeratin-19.

A

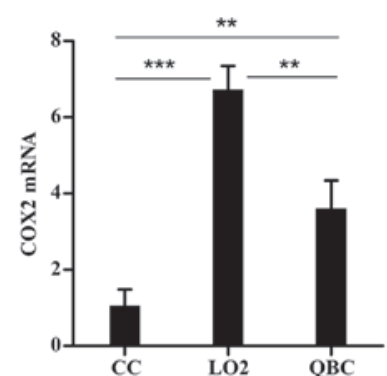

C
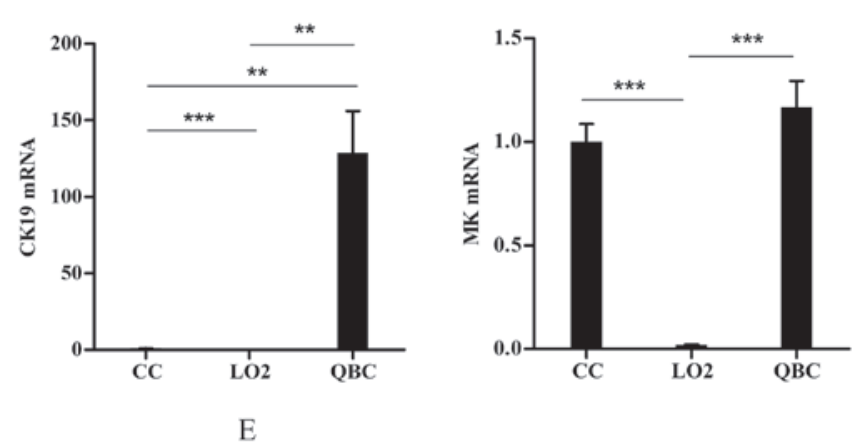

E

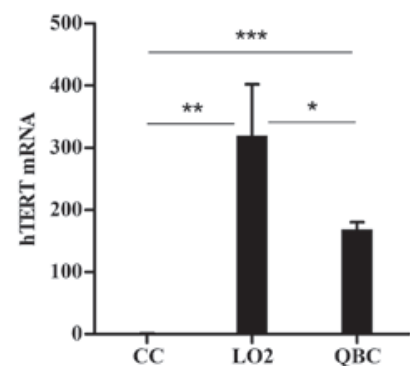

Figure 1. Analysis of mRNA expression of tumor biomarker genes in primary human cholangiocytes, primary human hepatocytes and cholangiocarcinoma cells. All mRNA expression was determined by reverse transcription-quantitative polymerase chain reaction and normalized to GAPDH. The expression level was calculated from $\mathrm{C}_{\text {quantification }}$ cycle $(\mathrm{Cq})$ values using the $2^{-\Delta \Delta C q}$ method and the expression levels were presented as the mRNA ratios of the cells analyzed to the primary human cholangiocytes. Data are expressed as mean \pm standard deviation. ${ }^{* * * *} \mathrm{P}<0.001,{ }^{* *} \mathrm{P}<0.01$ and ${ }^{*} \mathrm{P}<0.05$. COX-2, cyclooxygenase-2; MUC1, mucin-1; CK19, cytokeratin-19; MK, midkine; hTERT, human telomerase reverse transcriptase; $\mathrm{CC}$, primary human cholangiocyte; LO2, primary human hepatocyte; QBC, QBC939 cholangiocarcinoma cell line.

Unexpectedly, the Ad-CK19-Luc activity in primary human hepatocytes was higher compared with that in primary human

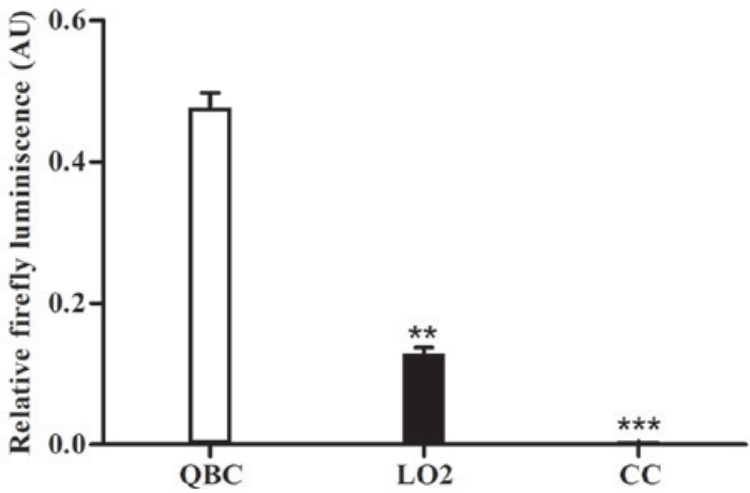

Figure 2. Activity of adenoviral vector constructed with CK19 promoter fragment. The fragment of the CK19 promoter was constructed to adenoviral vector pAdeno. The resulting adenoviral reporter vector Ad-CK19-Luc was transduced in triplicate to cholangiocarcinoma cells, primary human hepatocytes and primary human cholangiocytes. The luciferase activity of specific constructs was presented as the percentage of Ad-CMV-Luc activity to Ad-CMV-Luc activity for each individual cell type. CK19, cytokeratin-19; $\mathrm{CC}$, primary human cholangiocyte; LO2, primary human hepatocyte; QBC, QBC939 cholangiocarcinoma cell line. ${ }^{* * *} \mathrm{P}<0.001,{ }^{* *} \mathrm{P}<0.01$.

cholangiocytes, and did not correlate with its lowest mRNA expression. The results indicated that the CK19 promoter fragment was highly active in human cholangiocarcinoma cells and remained inactive in primary human hepatocytes and cholangiocytes; therefore, it may be a candidate TSP of choice in gene therapy for cholangiocarcinoma.

\section{Discussion}

Cholangiocarcinoma is associated with high mortality, rapidly increasing incidence and limited effectiveness of surgical therapy (15). Gene-based therapy appears to be a viable option for the treatment of certain highly malignant tumors, such as cholangiocarcinoma (5). However, a major disadvantage lies in the hepatotropism of the delivered vector, which is associated with side effects and limits its potential clinical application. The adenoviral vectors constructed with TSPs can effectively restrict gene expression in tumor cells, as they conditionally replicate in these cells, and this may shed light to cancer gene therapy (16). It was reported that the use of survivin promoter in adenovector-mediated gene delivery restricted gene expression of the delivered gene only in melanoma cells (17). The results from another study demonstrated that prostate-specific antigen promoter-driven gene delivery 
demonstrated selective expression of the delivered genes only in prostate cancer cells (18). However, transcriptional targeting is only one of the approaches that specifically deliver genes in tumor cells. Other targeting approaches, such as cell cycle-specific promoters, treatment-responsive promoters, or dual-specificity promoters selective to both tumor and tissue, were also tested in gene therapy (19). In this case, the promoters of the genes of cholangiocarcinoma-specific biomarkers serve as potential TSPs to be used for cholangiocarcinoma gene therapy. In this regard, certain established biomarkers, such as COX-2, MK, MUC1, hTERT and the bile duct marker CK19 appear to be promising candidates, as there were tumor-specifically upregulated (13). However, the results on cholangiocarcinoma-specific promoters have been inconsistent. Therefore, the aim of the present study was to identify TSPs which may be used for the development of adenoviral gene therapy for cholangiocarcinoma.

Our results demonstrated that CK19 gene expression displayed the best specificity to cholangiocarcinoma, as indicated by the significant difference in the mRNA expression of CK19 in cholangiocarcinoma cells, primary human hepatocytes and cholangiocytes. Although the mRNA expression of COX-2, hTERT and MUC1 was pronounced in cholangiocarcinoma cells, it was markedly lower compared with that in primary human hepatocytes, excluding the use of these genes for the selection of cholangiocarcinoma-specific promoters. The mRNA expression of MK was high in both cholangiocarcinoma cells and cholangiocytes; therefore, it was not suitable as a candidate TSP for cholangiocarcinoma. It should be noted that our results are partially inconsistent with those from a previous study, which reported that the mRNA expression of both hTERT and CK19 genes in cholangiocarcinoma cells was significantly higher compared with that in normal primary human hepatocytes (13). This inconsistency may be explained by the fact that the previous study used a different cholangiocarcinoma cell line, CC-LP-1.

As the vector components of the adenovirus may affect promoter activity, it is necessary to evaluate the promoter activity of the CK19 fragment constructed in the Ad5 vector. The results revealed that, not only did the vector retain the substantial promoter activity of CK19, but it also displayed excellent tumor specificity compared with the corresponding luciferase activity in hepatocytes and cholangiocytes. The results from mRNA expression and promoter activity measurements suggested that the promoter of the CK19 gene can be used for adenovirus-based gene delivery in cholangiocarcinoma cells to effectively minimize adenoviral replication in normal liver and bile duct cells. It may be inferred that the low expression of Ad-CK19-Luc activity in primary hepatocytes and cholangiocytes is most likely due to a lack of specific promoters. By contrast, CK19 is a known bile duct marker. However, in certain cholestatic liver diseases, such as primary biliary cirrhosis or primary sclerosing cholangitis, cholangiocytes may have an activated (pro-proliferative) phenotype, which displays a higher than normal CK19 activity. Under these conditions, when the patients receive TSP-directed gene therapy, whether the activity of CK19 is sufficiently high to interfere with the physiology of normal cholangiocytes requires further investigation. It should be emphasized that there were distinct differences in CK19 promoter activity among different cells, so that it was consid- ered adequate for the development of adenoviral gene therapy for cholangiocarcinoma, as suggested by similar previously reported findings $(20,21)$. The data presented herein suggest that the promoter of the CK19 gene is a promising tumor-specific regulatory element and may be used to restrict the transcription of delivered genes in the treatment of cholangiocarcinoma.

\section{Acknowledgements}

We thank the National Natural Science Foundation of China for its support.

\section{Funding}

The present study was supported by the Major Project of National Natural Science Foundation of China (grant no. 81370561).

\section{Availability of data and materials}

The datasets used and/or analyzed during the current study are available from the corresponding author on reasonable request.

\section{Authors' contributions}

JQ designed the study, analyzed the data and was a major contributor in writing the manuscript. MW performed cell culture experiments. Jun Qin worked on the transfection of plasmids. QC performed PCR experiments. ZP analyzed the data and revised the manuscript. All authors read and approved the final manuscript.

\section{Ethics approval and consent to participate}

Not applicable.

\section{Patient consent for publication}

Not applicable.

\section{Competing interests}

The authors declare no conflict of interests related to the publication of this study.

\section{References}

1. Sirica AE: Cholangiocarcinoma: Molecular targeting strategies for chemoprevention and therapy. Hepatology 41: 5-15, 2005.

2. Patel T: Increasing incidence and mortality of primary intrahepatic cholangiocarcinoma in the United States. Hepatology 33: 1353-1357, 2001.

3. Taylor-Robinson SD, Foster GR, Arora S, Hargreaves S and Thomas HC: Increase in primary liver cancer in the UK, 1979-94. Lancet 350: 1142-1143, 1997.

4. Seo E, Abei M, Wakayama M, Fukuda K, Ugai H, Murata T, Todoroki T, Matsuzaki Y, Tanaka N, Hamada H and Yokoyama KK: Effective gene therapy of biliary tract cancers by a conditionally replicative adenovirus expressing uracil phosphoribosyltransferase: Significance of timing of 5-fluorouracil administration. Cancer Res 65: 546-552, 2005.

5. Nakano K, Todo T, Chijiiwa K and Tanaka M: Therapeutic efficacy of G207, a conditionally replicating herpes simplex virus type 1 mutant, for gallbladder carcinoma in immunocompetent hamsters. Mol Ther 3: 431-437, 2001. 
6. Schauer RJ, Meyer G, Baretton G, Schildberg FW and Rau HG Prognostic factors and long-term results after surgery for gallbladder carcinoma: A retrospective study of 127 patients. Langenbecks Arch Surg 386: 110-117, 2001.

7. Hayashi N, Yamamoto H, Hiraoka N, Dono K, Ito Y, Okami J, Kondo M, Nagano H, Umeshita K, Sakon M, et al: Differential expression of cyclooxygenase-2 (COX-2) in human bile duct epithelial cells and bile duct neoplasm. Hepatology 34: 638-650, 2001.

8. Itoi T, Shinohara Y, Takeda K, Nakamura K, Shimizu M, Ohyashiki K, Hisatomi H, Nakano H and Moriyasu F: Detection of telomerase reverse transcriptase mRNA in biopsy specimens and bile for diagnosis of biliary tract cancers. Int J Mol Med 7: 281-287, 2001

9. Kagaya M, Kaneko S, Ohno H, Inamura K and Kobayashi K Cloning and characterization of the 5'-flanking region of human cytokeratin 19 gene in human cholangiocarcinoma cell line. J Hepatol 35: 504-511, 2001.

10. Kato M, Shinozawa T, Kato S, Endo K and Terada T: Increased midkine expression in intrahepatic cholangiocarcinoma: Immunohistochemical and in situ hybridization analyses. Liver 20: 216-221, 2000.

11. Tamada S, Goto M, Nomoto M, Nagata K, Shimizu T, Tanaka S, Sakoda K, Imai K and Yonezawa S: Expression of MUC1 and MUC2 mucins in extrahepatic bile duct carcinomas: Its relationship with tumor progression and prognosis. Pathol Int 52: 713-723, 2002.

12. Afford SC and Young LS: Gene therapy for hepatocellular carcinoma-teaching old dogs new tricks. Hepatology 34: 207-209, 2001.

13. Lie-A-Ling M, Bakker CT, Deurholt T, Hoekstra R, Wesseling JG, Afford SC and Bosma PJ: Selection of tumour specific promoters for adenoviral gene therapy of cholangiocarcinoma. J Hepatol 44: 126-133, 2006

14. Nagi P, Vickers SM, Davydova J, Adachi Y, Takayama K, Barker S, Krasnykh V, Curiel DT and Yamamoto M: Development of a therapeutic adenoviral vector for cholangiocarcinoma combining tumor-restricted gene expression and infectivity enhancement. J Gastrointest Surg 7: 364-371, 2003.

15. Jarnagin WR: Cholangiocarcinoma of the extrahepatic bile ducts. Semin Surg Oncol 19: 156-176, 2000.

16. Saukkonen K and Hemminki A: Tissue-specific promoters for cancer gene therapy. Expert Opin Biol Ther 4: 683-696, 2004.
17. Lu B, Makhija SK, Nettelbeck DM, Rivera AA, Wang M, Komarova S, Zhou F, Yamamoto M, Haisma HJ, Alvarez RD, et al: Evaluation of tumor-specific promoter activities in melanoma. Gene Ther 12: 330-338, 2005.

18. Latham JP, Searle PF, Mautner V and James ND: Prostate-specific antigen promoter/enhancer driven gene therapy for prostate cancer: Construction and testing of a tissue-specific adenovirus vector. Cancer Res 60: 334-341, 2000.

19. Nettelbeck DM, Jérôme V and Müller R: Gene therapy: Designer promoters for tumour targeting. Trends Genet 16: $174-181,2000$.

20. Kurihara T, Brough DE, Kovesdi I and Kufe DW: Selectivity of a replication-competent adenovirus for human breast carcinoma cells expressing the MUC1 antigen. J Clin Invest 106: 763-771, 2000.

21. Wirth T, Zender L, Schulte B, Mundt B, Plentz R, Rudolph KL, Manns M, Kubicka S and Kühnel F: A telomerase-dependent conditionally replicating adenovirus for selective treatment of cancer. Cancer Res 63: 3181-3188, 2003. 